\title{
Zero-temperature Glauber dynamics on small-world networks
}

\author{
Carlos P. Herrero \\ Instituto de Ciencia de Materiales de Madrid, Consejo Superior de Investigaciones \\ Cientificas (CSIC), Campus de Cantoblanco, 28049 Madrid, Spain
}

(Dated: August 21, 2018)

\begin{abstract}
The zero-temperature Glauber dynamics of the ferromagnetic Ising model on small-world networks, rewired from a two-dimensional square lattice, has been studied by numerical simulations. For increasing disorder in finite networks, the nonequilibrium dynamics becomes faster, so that the ground state is found more likely. For any finite value of the rewiring probability $p$, the likelihood of reaching the ground state goes to zero in the thermodynamic limit, similarly to random networks. The spin correlation $\xi(r)$ is found to decrease with distance as $\xi(r) \sim \exp (-r / \lambda), \lambda$ being a correlation length scaling with $p$ as $\lambda \sim p^{-0.73}$. These results are compared with those obtained earlier for addition-type small world networks.

PACS numbers: 64.60.De, 05.50.+q, 05.70.Ln, 89.75.Hc
\end{abstract}

\section{INTRODUCTION}

In recent years, researchers have accumulated evidence that various kinds of complex systems can be described in terms of networks or graphs, where nodes play the role of system units and edges represent interactions between connected pairs of units. Thus, complex networks have been used to model several types of real-life systems, and to study various processes taking place on them [1, 2, 3, 4, 5]. In this context, some models of networks have been designed to explain empirical data in several fields (sociology, economy, biology, technology), as is the case of the so-called small-world networks, introduced by Watts and Strogatz in 1998 [6].

This kind of small-world networks are well suited to describe properties of systems with underlying topological structure ranging from regular lattices to random graphs [7, 8], by changing a single parameter [9]. They consist of a regular lattice, in which a fraction $p$ of the bonds between nearest-neighbor nodes are replaced by new random links, thus creating long-range "shortcuts" [6, 9]. This procedure generates networks in which one finds at the same time a local neighborhood (as in regular lattices) and some global properties of random graphs, as a small mean distance between pairs of sites. This short global length scale has been found to be relevant for several statistical physical problems on small-world networks, such as spread of infections [10, 11], signal propagation [6, 12, 13, 14], and information spreading [15, 16, 17, 18, 19, 20].

Cooperative phenomena in this kind of networks display unusual characteristics, associated to their peculiar topology [21, 22, 23, 24, 25, 26, 27]. Thus, a paramagnetic-ferromagnetic phase transition of meanfield type at finite temperature was found for the Ising model on small-world networks derived from onedimensional (1d) 21, 28, 29], as well as from $2 \mathrm{~d}$ and $3 \mathrm{~d}$ regular lattices [30, 31].

While the single-spin-flip Glauber dynamics at finite temperatures reaches the state given by thermodynamic equilibrium, the situation at zero temperature is not so clear. In fact, it has been shown that nontrivial phenomena appear, even for regular lattices [32, 33, 34, 35]. In $d=1$ one reaches the ordered ground state, but for higher dimensions the system may get stuck in a frozen state with domains of opposite magnetization 35]. The Glauber dynamics has been also investigated on various types of complex networks, in particular in smallworld networks [36, 37], Erdös-Rényi random graphs [38, 39, 40], and scale-free networks [39, 40, 41, 42]. In these cases, the system may become trapped in a set of ordered domains, without reaching a fully ordered state, even at finite system size. Analytical calculations, in particular, have been carried out to study several characteristics of this zero-temperature dynamics on regular lattices [32, 33, 43, 44] and complex networks [38, 39, 42]. A comparison with numerical simulations has shown that mean-field-type descriptions may be inappropriate to describe this problem [42].

Here we consider the zero-temperature Glauber dynamics in small-world networks rewired from a $2 \mathrm{~d}$ square lattice. This allows us to study the evolution of the system from a regular lattice to a random network by changing the rewiring probability $p$. Earlier works considered networks in which links were added to the regular lattice, so that a random network was not approached in the limit of large disorder [36, 37]. We will look for similarities and differences between the spin dynamics on these "addition-type" networks and those studied here. A question of particular interest is whether for small disorder $(p \ll 1)$ the system behaves like a random network, or on the contrary it keeps some characteristics of the regular lattice.

The paper is organized as follows. In Sec. II we describe the model employed in the paper. In Sec. III we discuss the ordering process, presenting results for the fraction of ordered and active runs, as well as the ordering time. In Sec. IV we give results for the active links, in Sec. V we present the spin correlation, and in Sec. VI we discuss the effect of the initial magnetization on the system evolution. The paper closes with a summary in Sec. VII. 


\section{MODEL}

To generate our networks, we start from a twodimensional square lattice. Contrary to the $1 \mathrm{~d}$ chain, it is known that in $2 \mathrm{~d}$ lattices the zero-temperature Glauber dynamics may get stuck in a frozen state without reaching the ground state (a ferromagnetic state, with all spins parallel), which makes the dynamics nontrivial [34, 35]. Small-world networks were built up according to the model of Watts and Strogatz [6, 9], i.e., we considered in turn each of the bonds in the starting $2 \mathrm{~d}$ lattice and replaced it with a given probability $p$ by a new connection. In this rewiring process, one end of the selected link is changed to a new node chosen at random in the whole network. We impose the conditions: (i) no two nodes can have more than one bond connecting them, (ii) no node can be connected by a link to itself, and (iii) each node has at least two connections. With this method we obtained networks where more than $99.9 \%$ of the sites were connected in a single component. Moreover, this rewiring procedure keeps constant the total number of links in the rewired networks, so that we have an average degree $\langle k\rangle=4$ irrespective of the rewiring probability $p$. This allows us to study the effect of disorder upon the properties of the model, without changing the mean connectivity. For networks generated in the present way there is a $p$-dependent crossover size that separates the large- and small-world regimes, and the small-world behavior appears for any finite value of $p(0<p<1)$ as soon as the network is large enough [45, 46]. All networks considered here are in the small-world regime [13, 30].

We note that other ways of generating small-world networks from regular lattices have been proposed [47, 48]. In particular, instead of rewiring each bond with probability $p$, one can add shortcuts between pairs of sites taken at random, without removing bonds from the regular lattice. This method turns out to be more convenient for analytical calculations, but does not keep constant the mean degree $\langle k\rangle$, which in this case increases with $p$. The zero-temperature Glauber dynamics of the Ising model has been studied earlier on these "addition-type" small-world networks. Thus, Boyer and Miramontes [36] studied interface motion and pinning in the limit $p \ll 1$, whereas Das and Sen [37] considered densely connected small-world networks, generated from one-dimensional rings.

From the 2d square lattice, we generated small-world networks of different sizes $N=L \times L$. For convenience, we will employ later $L$ instead of $N$ to indicate the system size in some plots. The largest networks used here included $N=400 \times 400$ nodes. We considered periodic boundary conditions for the starting regular lattice, and then the rewiring process was carried out in a way similar to that described in Ref. [6]. Each network is characterized by its adjacency matrix $A_{i j}$, where $A_{i j}=1$ if nodes $i$ and $j$ are connected by a link, and $A_{i j}=0$ otherwise. For a given network, we consider an Ising model with spin variables $S_{i}= \pm 1(i=1, \ldots, N)$ located on the nodes of the network, i.e., we have a Hamiltonian:

$$
H=-\sum_{i<j} A_{i j} S_{i} S_{j} .
$$

This means that each edge in the network represents a ferromagnetic interaction between spins on the two linked nodes. The spin configuration evolves in time, and at a given time $t$, each site $i$ experiences a local field $h_{i}(t)$ due to the spins located at its nearest-neighbor nodes:

$$
h_{i}(t)=\sum_{j} A_{i j} S_{j}(t) .
$$

The zero-temperature Glauber dynamics is then defined as follows. At time step $t+1$ one selects at random a node from a uniform probability distribution, i.e. all nodes are equally likely to be selected, irrespective of the particular properties of each one, such as its degree $k$. Note that other ways of choosing the nodes, as a deterministic sweep, or according to a degree-dependent probability distribution, introduce an undesired bias in the spin dynamics. Once a node is selected, the value of its spin is updated according to the local field, namely:

$$
S_{i}(t+1)= \begin{cases}+1 & \text { if } h_{i}(t)>0 \\ -1 & \text { if } h_{i}(t)<0 \\ \pm 1 & \text { with probability } \frac{1}{2} \text { if } h_{i}(t)=0\end{cases}
$$

We will measure time as the number of attempted updates per node, $N_{s}=t / N$ (simulation sweeps), so that on average each node tries to change its state once per simulation sweep. Several variables characterizing the considered model have been calculated and averaged for different values of $p$. In general, we have considered 1000 simulation runs for each rewiring probability $p$, but in some cases we carried out up to 5000 runs to improve the precision of our results, in particular to determine the average number of active links (see below). We started the simulations by assigning to each node a spin +1 or -1 with $50 \%$ probability. In Sect. V, however, we consider the effect of having a starting finite magnetization on the evolution of the system. For each run considered, the simulation proceeds while the energy of the system is changing, and stops when it is constant for a long simulation interval. This interval is taken to be equal to $2000 N$ attempted spin updates, i.e., 2000 simulation sweeps. This is what we will call in the following the infinite-time limit, to distinguish it from the simulation times at which the system energy is still changing.

\section{ORDERING PROCESS}

For the zero-temperature Glauber dynamics on the $2 \mathrm{~d}$ square lattice, it is known that the system reaches either a frozen stripe state with probability $\approx 1 / 3$ or the ordered ground state with probability $\approx 2 / 3[35]$. For small-world networks we find that the fraction of ordered configurations depends markedly on the rewiring 


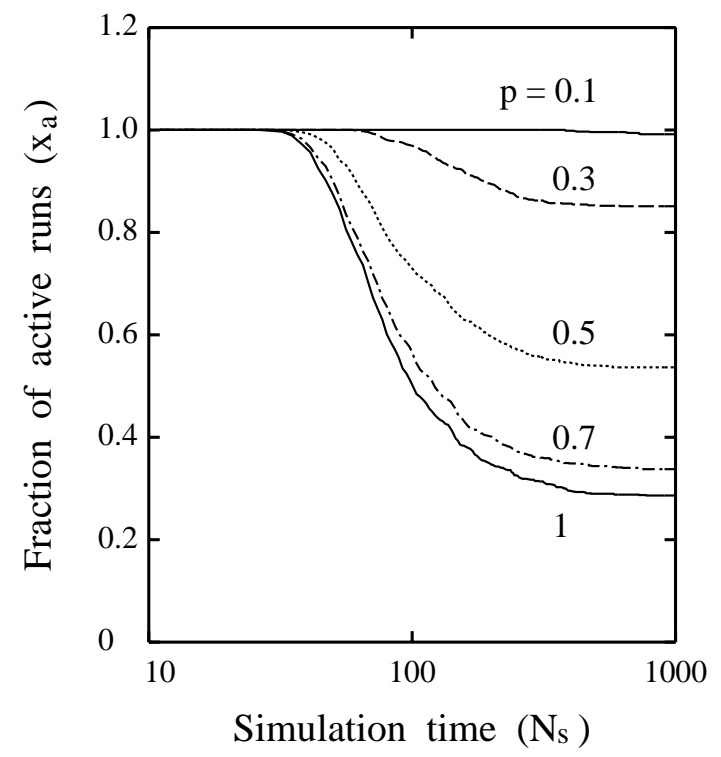

FIG. 1: Fraction of active spin configurations vs. number of simulation sweeps, $N_{s}$, for several values of the rewiring probability $p$. From top to bottom: $p=0.1,0.3,0.5,0.7$, and 1. Data correspond to simulations on networks with 6400 nodes.

probability. We will call active runs those that have not reached the ground state at a given simulation time. For a given parameter set $(p, N)$ we will denote the fraction of active runs by $x_{a}$. In Fig. 1 we display $x_{a}$ as a function of the number of simulation sweeps, for several values of $p$. These results were obtained by averaging in each case over 1000 spin configurations on networks including 6400 nodes. From these results we observe that the system gets ordered more frequently as the rewiring probability increases. In fact, for $p=0.1$ we find at large simulation times a fraction of active spin configurations larger than 0.99, i.e. a fraction of ordered configurations less than 0.01. By comparing with the known result for the regular lattice $(p=0)$, this means that a small fraction of rewired links is enough to keep the system with a large probability in a disordered configuration. This is in line with the result found in Ref. 36 for addition-type smallworld networks, in the sense that nodes with long-range connections strongly affect the motion of interfaces and thus inhibit reaching the ground state.

For larger $p$ the underlying regular lattice is progressively destroyed, approaching a random network in the limit $p \rightarrow 1$. For random networks it is known that the long-time behavior of the model is very different from that of the $2 \mathrm{~d}$ square lattice. For such networks the probability of the system reaching the ground state goes to zero in the large-network limit $(N \rightarrow \infty)$ 38]. In Fig. 1 one also observes that the typical ordering time for smallworld networks decreases as the rewiring probability is raised. This is general for different network sizes, as will be discussed below.

For finite networks, it is interesting to check the

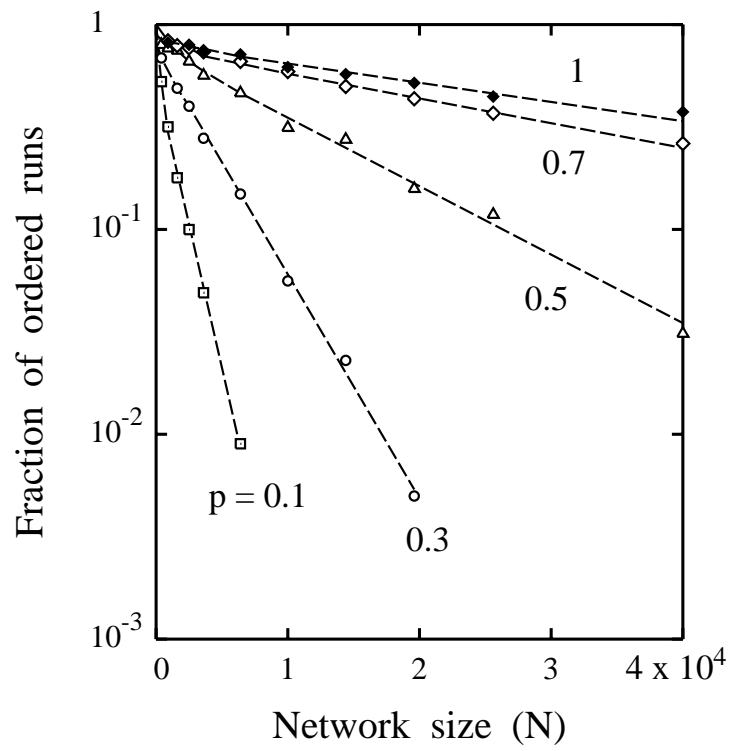

FIG. 2: Fraction of simulation runs reaching the ground state vs. network size $N$ in the limit of infinite simulation time. Each kind of symbols indicates a rewiring probability $p$. From top to bottom: $p=1,0.7,0.5,0.3$, and 0.1 . Lines are guides to the eye. Error bars are on the order of the symbol size.

crossover from the properties of the regular lattice to those characterizing the small-world regime. This crossover will happen at a rewiring probability $p_{c}$ that depends on the system size, and decreases as the system size rises (for $N \rightarrow \infty, p_{c} \rightarrow 0$ ). Thus, for the system size $N=6400$ considered in Fig. 1, one has a change in the fraction of active networks at large time, $x_{a}$, from $\approx 1 / 3$ for the square lattice $(p=0)$ to 0.99 for $p=0.1$. This means that there is a fast change of $x_{a}$ in this region, due to the onset of the small-world regime. We have checked that, in fact, the crossover appears for this system size at $p_{c} \approx 0.02$.

In Fig. 2 we show the fraction of ordered configurations, $1-x_{a}$, as a function of the network size $N$, in the limit of large simulation time. We display in a semilogarithmic plot results for various values of the rewiring probability, between $p=0.1$ and $p=1$. In all cases the probability of a spin configuration reaching the ground state decreases exponentially as the network size increases, and the system remains trapped in spin configurations with part of the nodes with $S_{i}=1$ and the rest with $S_{i}=-1$. This behavior is similar to that observed for the Glauber dynamics in random networks at $T=0$. In fact, Svenson [49] has noticed a freezing in a disordered state for Glauber dynamics on random graphs. This problem was considered analytically by Häggström, who showed that the dynamics fails to reach the ordered ground state in random networks in the large-size limit $N \rightarrow \infty$ [38]. It is important to emphasize the change in $x_{a}$ from the regular lattice to small-world networks in the thermodynamic limit. In fact, $x_{a}$ changes from $\approx 1 / 3$ for the square lattice $(p=0)$ to 1 for any rewiring probability 


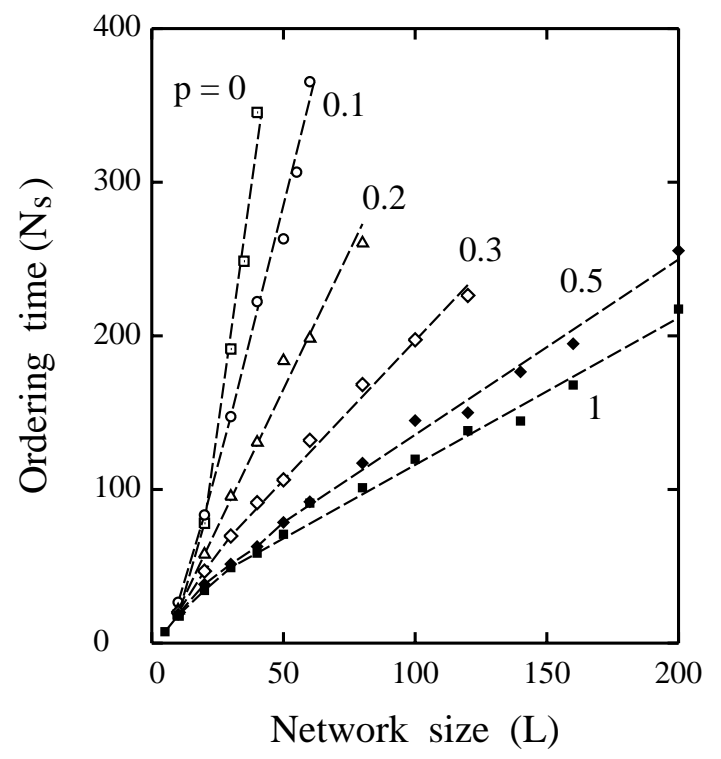

FIG. 3: Mean ordering time as a function of network size for several values of the rewiring probability $p$. Symbols indicate results derived from zero-temperature simulations. From left to right: $p=0,0.1,0.2,0.3,0.5$, and 1 .

$>0$. The small-world topology thus precludes the system from reaching the ordered ferromagnetic state in the large-size limit. The similarity of our results for rewired small-world networks with those found for random networks is a difference with addition-type networks, since in the latter the connections in the underlying regular lattice are preserved for any value of $p$, and one does not recover the behavior of random networks.

Another interesting quantity is the mean ordering time of the system, $N_{o}$. It is defined as the average simulation time employed by the system to reach a ground-state configuration, calculated for the runs that actually become ordered. The mean ordering time is displayed in Fig. 3 as a function of the network size for several values of $p$, between 0 and 1 . For a given rewiring probability, $N_{o}$ increases linearly with $L$, i.e. $N_{o} \sim \sqrt{N}$. For a given network size, the system gets ordered faster for larger $p$. This means that for small $p$ there appear less ordered configurations (see Fig. 2), and those that appear take longer times to reach the ground state. This is an interesting difference between the behavior of $x_{a}$ and $N_{o}$ for small $p$. For a given network size, $N_{o}$ evolves in a continuous manner from $p=0$ to $p=1$, as shown in Fig. 3 . However, the fraction of active runs $x_{a}$ for $p=0$ behaves in a way different from that for $p>0$, since in the former case $x_{a}$ converges to $\approx 1 / 3$ as the network size increases [35, 50], and in the latter $x_{a} \rightarrow 1$ in the large-size limit (see above).

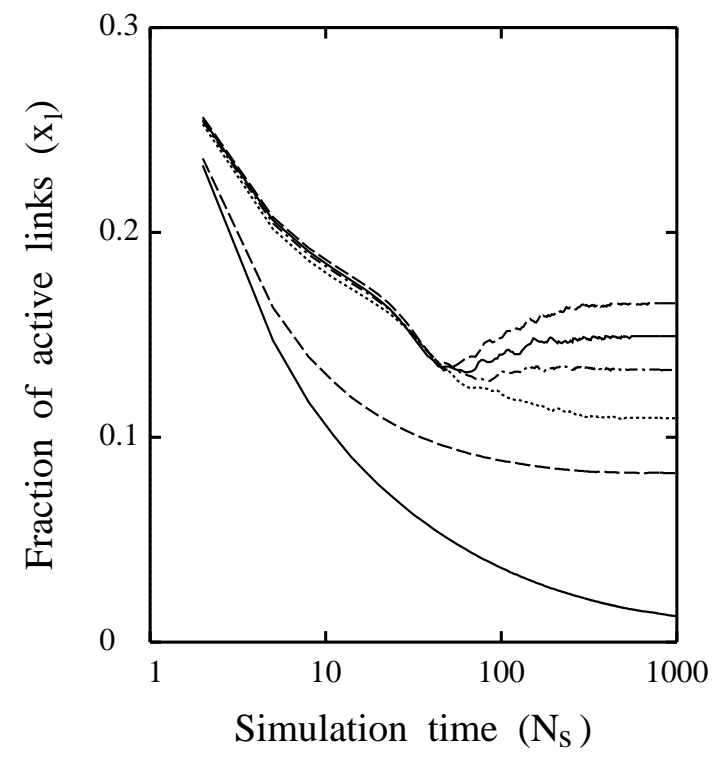

FIG. 4: Fraction of active links in active runs as a function of the number of simulation sweeps. Lines correspond to various values of the rewiring probability $p$. From top to bottom: $p=1,0.7,0.6,0.5,0.1$, and 0 . These data correspond to simulations on networks with 6400 nodes.

\section{ACTIVE LINKS}

The failure of the system to reach an ordered state for system size $N \rightarrow \infty$ could be due to the presence of small clusters (communities) of nodes tightly connected with each other, but loosely linked to the rest of the network. Such clusters could become ordered independently of the rest of the system, thus giving a disordered state, and hindering the reach of the ground state. This is the mechanism discussed in Ref. 39 in connection with the Glauber dynamics on random networks. To address the validity of this assumption, it is useful to consider the active links in a network, defined as those connecting nodes with opposite values of $S_{i}$. In the following we will call $x_{l}$ the fraction of active links, averaged over active runs. The evolution of $x_{l}$ along the simulation runs is presented in Fig. 4 for various values of the rewiring probability and networks including 6400 nodes. We note first that, for a given simulation time $N_{s}$, the fraction of active links increases as $p$ is raised. For $p \leq 0.5, x_{l}$ decreases as time proceeds and eventually converges to a finite value. For $p>0.5$ we find a decrease in $x_{l}$ at short times, reaching a minimum for $N_{s} \sim 100$, and rising latter to saturate to a finite value at large times. In principle, for the ordering process advancing as time proceeds, the number of actives links in active configurations decreases, as found for $p<0.5$. However, a fast decrease in the fraction of active runs can cause a rise in $x_{l}$, since the latter is calculated over runs still active, and those getting ordered are expected to have few active links. In fact, the minimum in $x_{l}$ observed for $p>0.5$ occurs at the same simulation times as the fast decrease in $x_{a}$ shown in Fig. 1 . This 


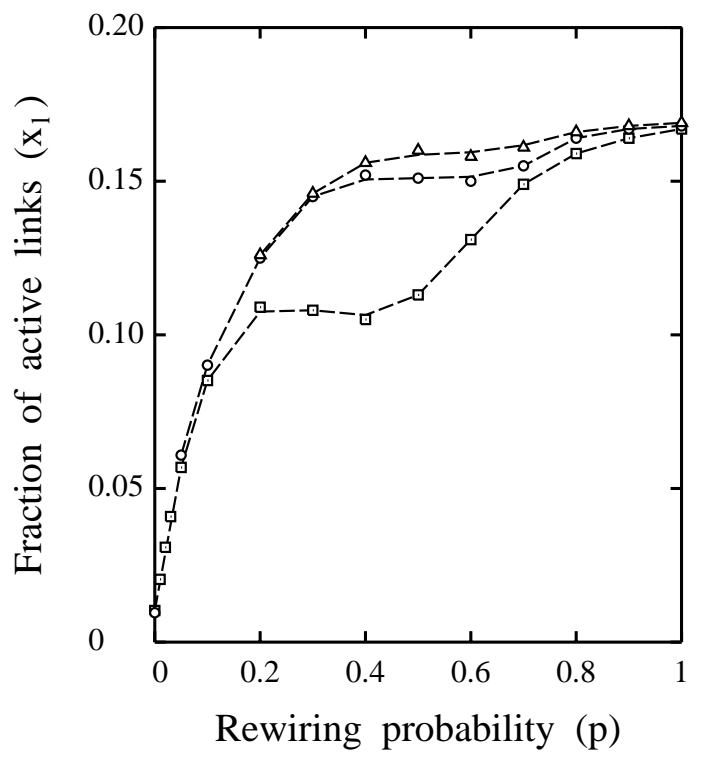

FIG. 5: Fraction of active links in active runs vs rewiring probability $p$, in the limit of infinite simulation time. Symbols represent results of simulations for networks with different sizes: squares, $L=100$; circles, $L=300$; triangles, $L=400$. Error bars are on the order of the symbol size. Lines are guides to the eye.

minimum in $x_{l}$ is reminiscent of that found by Castellano et al. 39] for random networks with average degree $\langle k\rangle=7$.

The long-time value of $x_{l}$ is presented in Fig. 5 as a function of the rewiring probability $p$ for three different system sizes. Symbols indicate results of our simulations: squares for $L=100$, circles for $L=300$, and triangles for $L=400$. For $L=100$ we find that $x_{l}$ increases with $p$, but has a plateau at intermediate values of the rewiring probability. This plateau, however, seems to be a finite-size effect, since it tends to disappear as the network size is increased. In fact, for $L=400$, it is almost inappreciable; one has a fast rise in the fraction of active links $x_{l}$ between $p=0$ and $p \approx 0.3$, and the rise is much slower for $p>0.4$. Finally, in the largedisorder limit $(p=1) x_{l}$ converges to a value of about 0.17 , irrespective of the system size $L$. Interestingly, a clear finite-size effect appears for intermediate values of $p$, but is very small in both limits $p \rightarrow 0$ (regular lattice) and $p \rightarrow 1$ (random networks). Close to $p=0$ we find a linear increase in $x_{l}$, with a slope $d x_{l} / d p=1.02 \pm 0.02$.

We now go back to the question posed above on the presence of small clusters of nodes that could get ordered independently of the rest of the network. If this was the reason for avoiding the full ordered state, one would expect to find a small fraction of active links, which should decrease for rising network size. This is not the case, as shown by our results displayed in Fig. 5. In line with this, we have also observed in our simulations that the long-time magnetization goes to zero when increasing the system size, as expected for the presence of different large

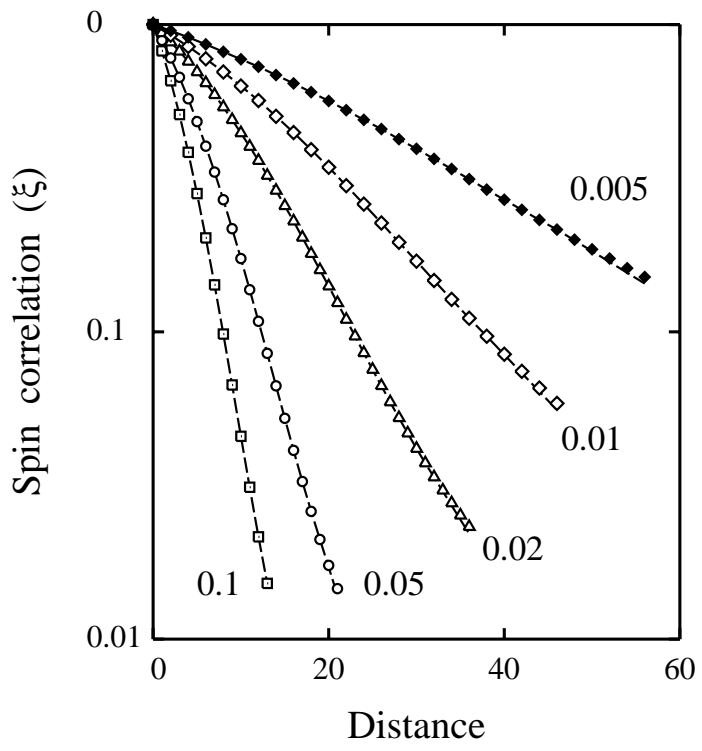

FIG. 6: Spin correlation $\xi(r)$ vs distance $r$ for active runs on small-world networks with $9 \times 10^{4}$ nodes, in the limit of large simulation time $N_{s} \rightarrow \infty$. Error bars are in the order of the symbol size.

ordered regions in the network. This conclusion agrees with that given in Ref. 39 for random networks, but here it extends to small-world networks in the whole range from $p=0$ to 1 .

In connection with this, it is important the question whether the disordered (metastable) state reached after long simulation time is a frozen state or not. In our simulations we find that the disordered state is active, in the sense that some spins continue flipping forever, without changing the energy of the system. Both spin states $(+1$ and -1$)$ coexist, with several connected domains of identical spins. The continuous flipping happens at the borders of these domains. This behavior is similar to that observed for regular lattices for $d>2$ and for random networks [39]. This means that a small fraction of rewired links $(p \ll 1)$ is enough to recover the behavior of random networks, instead of the freezing observed for the Glauber dynamics on the $2 \mathrm{~d}$ square lattice [35].

\section{SPIN CORRELATION}

From the results presented above, it is clear that the fraction of ordered configurations on small-world networks goes to zero in the large-size limit, and thus the ground state is not reached in the thermodynamic limit. A direct way of displaying the lack of long-range ferromagnetic ordering is by looking at the spin correlation vs distance for various values of the rewiring probability $p$. We define $\xi$ as

$$
\xi(r)=\left\langle S_{i} S_{j}\right\rangle_{r},
$$




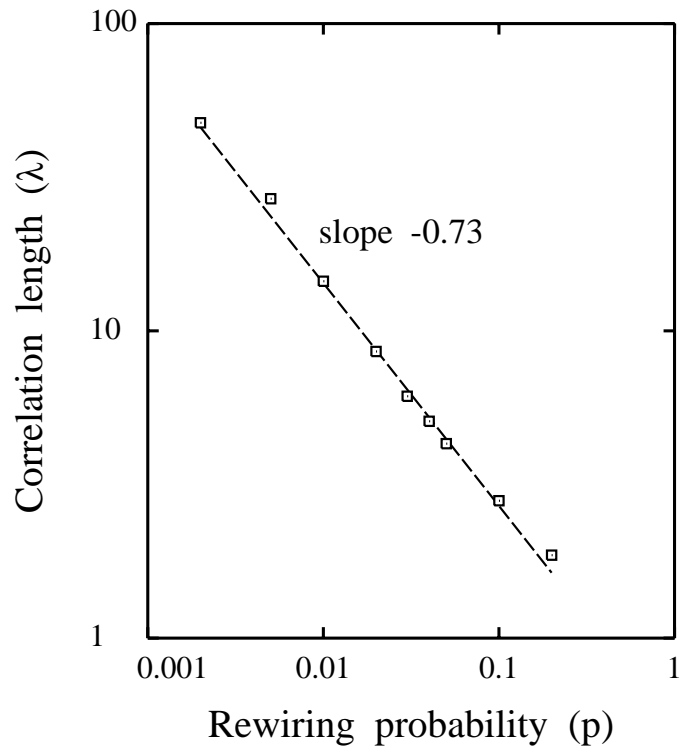

FIG. 7: Correlation length $\lambda$ vs rewiring probability in a logarithmic plot. Data points were obtained from the decay of the spin correlation $\xi(r)$ with distance, for networks including $9 \times 10^{4}$ nodes, in the limit of large simulation time $N_{s} \rightarrow \infty$. Error bars are on the order of the symbol size.

where the subscript $r$ indicates that the average is taken for the ensemble of pairs $(i, j)$ of sites at distance $r$. Note that $r=d / d_{0}$ refers here to the dimensionless distance between sites in the starting regular lattice, not to the actual topological distance or minimum number of links between nodes in the rewired networks $\left(d_{0}\right.$ is the distance between nearest neighbors). The correlation $\xi(r)$ in the long-time limit is shown in Fig. 6 for several values of the rewiring probability $p$. After a short transient for small $r, \xi(r)$ displays a logarithmic decrease for increasing distance. At distances longer than those presented in Fig. 6, $\xi(r)$ saturates to a constant value, which is a finite-size effect and should disappear in the limit $N \rightarrow \infty$. Note that the results displayed in Fig. 6 correspond to values of the rewiring probability $p \ll 1$. For larger $p$, the decrease in $\xi(r)$ as a function of distance is very fast.

According to the results shown in Fig. 6, we find a region where the spin correlation scales as $\xi(r) \sim$ $\exp (-r / \lambda)$, with a correlation length $\lambda$ dependent on the rewiring probability $p$. This dependence of $\lambda$ on $p$ is shown in Fig. 7 in a logarithmic plot, where one observes that the correlation length follows over two decades a power law $\lambda \sim p^{-a}$, with an exponent $a=0.73 \pm 0.02$.

Boyer and Miramontes [36] interpreted the presence of different spin domains on their addition-type smallworld networks in terms of the "influent" nodes. These are nodes that have long-range connections, and strongly affect the motion of domain interfaces. Moreover, they argued that the exponent $a$ should be $2 / 3$, on the basis of the random distribution of influent nodes over the square lattice. In fact, they found a value $a=0.64$ from their numerical simulations, close to $2 / 3$. The exponent

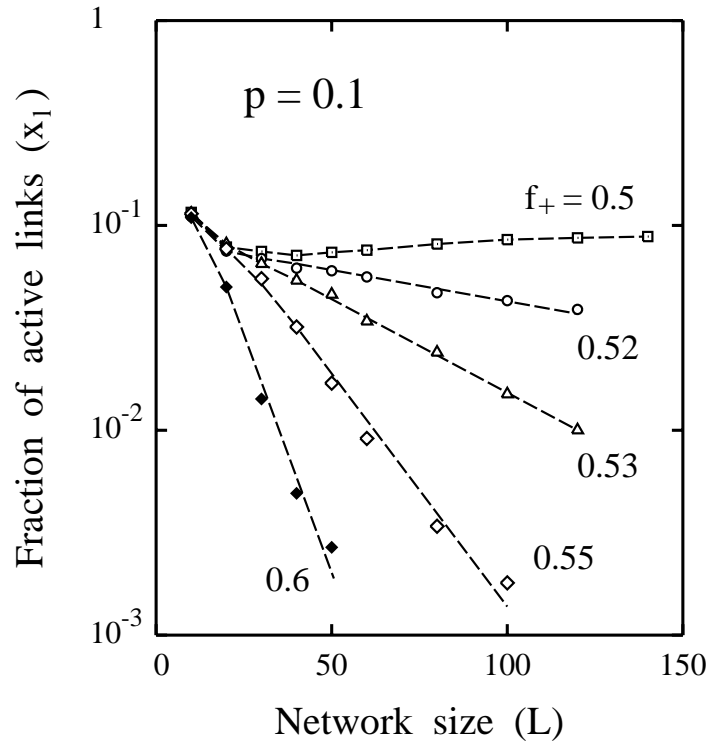

FIG. 8: Fraction of active links vs network size for several values of the initial magnetization. Symbols indicate results derived from simulations for a rewiring probability $p=0.1$, in the limit of infinite simulation time. From top to bottom: $f_{+}=0.5,0.52,0.53,0.55$, and 0.6. For $f_{+}>0.6, x_{l}$ decreases very fast for increasing network size (not shown).

yielded by our simulations on rewired networks is somewhat higher. This can be understood by taking into account that in our case the influent nodes play a role similar to that played in addition-type small-world networks, but also connections in the underlying lattice are progressively destroyed as the rewiring probability increases. This affects the correlation between sites in the lattice, which is effectively reduced and the exponent $a$ increases with respect to that expected for the full square lattice $(\lambda$ decreases faster for rising $p)$.

\section{INFLUENCE OF INITIAL CONDITIONS}

The results shown above were obtained for initial spin configurations with zero average magnetization (spins with $50 \%$ probability for +1 and -1 ). One can also consider starting spin configurations with different probabilities for spins +1 and -1 , and study the evolution of the system under the Glauber dynamics at $T=0$. A study of the effect of initial conditions on this type of dynamics in various kinds of complex networks has been carried out earlier by Uchida and Shirayama [40]. These authors emphasized that both the initial conditions and the network structure are equally relevant to determine the evolution of the system. This evolution may be nontrivial, mainly in the presence of nodes with large degree, as happens in scale-free networks.

We now consider initial configurations on our smallworld networks, where the probability $f_{+}$of $S_{i}=+1$ is higher than 0.5 , and study their evolution with time. In 


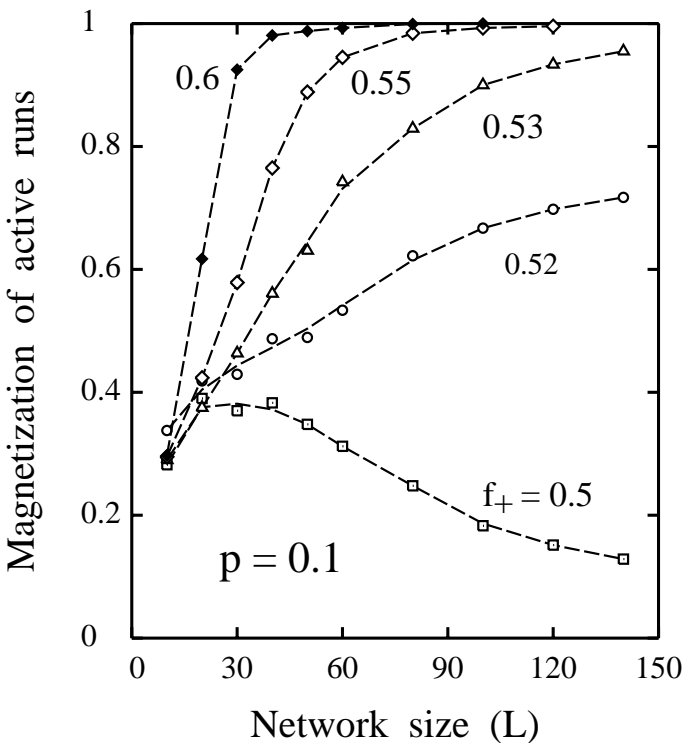

FIG. 9: Average magnetization $\bar{M}$ of spin configurations in active runs as a function of network size, for various values of the initial magnetization. Symbols show results derived from simulations on networks with a rewiring probability $p=0.1$, in the limit of infinite simulation time. From top to bottom: $f_{+}=0.6,0.55,0.53,0.52$, and 0.5 . For $f_{+}>0.6, \bar{M}$ converges fast to 1 for increasing network size (not shown). Dashed lines are guides to the eye.

Fig. 8 we present the fraction of active links as a function of the network size for several values of $f_{+}$between 0.5 and 0.6. These results were obtained for a rewiring probability $p=0.1$ and in the long-time limit $N_{s} \rightarrow \infty$. For $f_{+}=0.5$ (random initial configuration), the fraction $x_{l}$ converges to a finite value as the network size increases, as shown above. For $f_{+}>0.5$, however, $x_{l}$ decreases exponentially as $N$ is raised, indicating that the system evolves to a single ordered domain, eventually converging to the ground state.

To check this point we have calculated the average magnetization $\bar{M}$ over active networks as a function of the system size. We define $\bar{M}$ as $\bar{M}=\langle|M|\rangle_{a c}$, with $M=\sum_{i} S_{i}$. In Fig. 9 we show $\bar{M}$ for the same rewiring probability and $f_{+}$values presented in Fig. 8. Again the results correspond to the long-time limit. For random initial conditions the average magnetization decreases and goes to zero for $N \rightarrow \infty$, as indicated above (for $L=300$, we find $\bar{M}=0.057$, not shown in the figure). On the contrary, for $f_{+}>0.5$, the average magnetization increases as the network size is raised, converging to the value expected for the fully ordered system $(\bar{M}=1)$. This is consistent with the decrease observed in the fraction of active bonds $x_{l}$ for rising $N$, shown in Fig. 8. Moreover, this behavior is reminiscent of that observed for random networks in Ref. 40, in the sense that a small finite value of the starting magnetization is enough to drive the system to an ordered state. Such an evolution can be different for other kinds of complex networks [40].

Uchida and Shirayama 40] found that, depending on the type of network, the final dynamics may reach a metastable state involving two coexistent spin states with several connected domains of identical spins, the marginal vertices of which flip continuously. This is in fact the behavior found for our small-world networks and presented above for random initial conditions $\left(f_{+}=0.5\right)$ in section IV. For $f_{+}>0.5$, however, we have found that the system converges to an ordered state in the thermodynamic limit $N \rightarrow \infty(\bar{M} \rightarrow 1)$, but has a finite probability of being disordered for any finite size. Both Figs. 8 and 9 indicate that, for a given value $f_{+}>0.5$, the appearance of a single spin domain is favored as the system size increases. However, the convergence to the ordered state for increasing system size is fast for $p>0.6$, but becomes very slow as one approaches the random initial conditions, and finally the system remains in a disordered state for $f_{+}=0.5$ and $N \rightarrow \infty$ (see above). Note that for $f_{+}>0.5$ the behavior of the system is opposite to that shown above for $f_{+}=0.5$, in the sense that for random initial conditions the system reaches the ground state less frequently for increasing size $N$ (see also the increase in $x_{l}$ shown in Fig. 8 for $f_{+}=0.5$ and decrease in $\bar{M}$ in Fig. 9, as $N$ rises).

\section{SUMMARY}

We have studied numerically the Glauber dynamics of the ferromagnetic Ising model on small-world networks, generated by rewiring links in a two-dimensional square lattice. We have found that the behavior of the model departs from that known for the regular lattice, even for a small fraction of rewired links. In fact, for any rewiring probability $p$, the fraction of ordered runs for large time $\left(N_{s} \rightarrow \infty\right)$ and large system size $(N \rightarrow \infty)$ goes to zero, contrary to the regular lattice, where this fraction converges to a finite value $(\approx 2 / 3)$. For finite networks, the system gets ordered more frequently as the rewiring probability increases and one approaches a random network.

The spin correlation on the underlying lattice is found to decrease as $\xi(r) \sim \exp (-r / \lambda)$, with a correlation length that depends on the rewiring probability as $\lambda \sim$ $p^{-0.73}$. The exponent giving the dependence of $\lambda$ on $p$ is close to but different from that derived earlier for addition-type small-world networks.

We have analyzed the influence of the initial conditions on the behavior of the system. We found that for any small deviation from random initial conditions, the system evolves to the ordered state in the limit of large system size and simulation time, contrary to the behavior obtained from random initial conditions.

The apparently simple Glauber dynamics at $T=0$ displays a nontrivial behavior, that depends upon the type of network on which it is defined. This is true even for regular lattices with $d>1$. For small-world networks rewired from $2 \mathrm{~d}$ lattices, we find a behavior of the Glauber dynamics similar to that corresponding to random networks. This refers in particular to the nature 
of the disordered state obtained at long times, which is characterized by the presence of ordered regions, with spins at the interfaces between domains flipping forever without changing the energy. This behavior is different from the frozen state reached for $2 \mathrm{~d}$ regular lattices, but is reminiscent of that for lattices with dimensionality larger than 2. An important result is that for our small-world networks, a small fraction of disorder (in fact any rewiring probability $p>0$ ) is enough to recover the behavior known for random networks.

For small-world networks generated from lattices with a dimensionality different from 2, we expect a behavior qualitatively similar to that presented here for $d=2$. However, the actual details of the nonequilibrium order- ing process on these networks may depend on $d$. Also, the behavior of the model for generalized random networks may display some characteristics different than ErdösRényi networks. These points require further investigation, and remain as a challenge for future research.

\section{Acknowledgments}

This work was supported by Ministerio de Ciencia e Innovación (Spain) under Contract No. FIS2006-12117C04-03.
[1] R. Albert and A. L. Barabási, Rev. Mod. Phys. 74, 47 (2002).

[2] S. N. Dorogovtsev and J. F. F. Mendes, Evolution of Networks: From Biological Nets to the Internet and $W W W$ (Oxford University, Oxford, 2003).

[3] M. E. J. Newman, SIAM Rev. 45, 167 (2003).

[4] M. E. J. Newman, A. L. Barabási, and D. J. Watts, eds., The structure and dynamics of networks (Princeton University, Princeton, 2006).

[5] L. da F. Costa, F. A. Rodrigues, G. Travieso, and P. R. Villas Boas, Adv. Phys. 56, 167 (2007).

[6] D. J. Watts and S. H. Strogatz, Nature 393, 440 (1998).

[7] B. Bollobás, Modern Graph Theory (Springer-Verlag, New York, 1998).

[8] D. S. Callaway, M. E. J. Newman, S. H. Strogatz, and D. J. Watts, Phys. Rev. Lett. 85, 5468 (2000).

[9] D. J. Watts, Small Worlds (Princeton University Press, Princeton, 1999).

[10] M. Kuperman and G. Abramson, Phys. Rev. Lett. 86, 2909 (2001).

[11] C. Moore and M. E. J. Newman, Phys. Rev. E 61, 5678 (2000).

[12] L. F. Lago-Fernández, R. Huerta, F. Corbacho, and J. A. Sigüenza, Phys. Rev. Lett. 84, 2758 (2000).

[13] C. P. Herrero, Phys. Rev. E 66, 046126 (2002).

[14] Y. Moreno, M. Nekovee, and A. F. Pacheco, Phys. Rev. E 69, 066130 (2004).

[15] J. Lahtinen, J. Kertész, and K. Kaski, Phys. Rev. E 64, 057105 (2001).

[16] S. A. Pandit and R. E. Amritkar, Phys. Rev. E 63, 041104 (2001).

[17] V. Latora and M. Marchiori, Phys. Rev. Lett. 87, 198701 (2001).

[18] C. P. Herrero and M. Saboyá, Phys. Rev. E 68, 026106 (2003).

[19] C. P. Herrero, Eur. Phys. J. B 56, 71 (2007).

[20] J. Candia, J. Stat. Mech.: Theory Exp. p. P09001 (2007).

[21] A. Barrat and M. Weigt, Eur. Phys. J. B 13, 547 (2000).

[22] M. E. J. Newman, J. Stat. Phys. 101, 819 (2000).

[23] C. Moore and M. E. J. Newman, Phys. Rev. E 62, 7059 (2000).

[24] P. Svenson and D. A. Johnston, Phys. Rev. E 65, 036105 (2002).
[25] J. Candia, Phys. Rev. E 74, 031101 (2006).

[26] S. N. Dorogovtsev, A. V. Goltsev, and J. F. F. Mendes, Rev. Mod. Phys. 80, 1275 (2008).

[27] C. P. Herrero, Phys. Rev. E 77, 041102 (2008).

[28] M. Gitterman, J. Phys. A: Math. Gen. 33, 8373 (2000).

[29] J. Viana Lopes, Y. G. Pogorelov, J. M. B. Lopes dos Santos, and R. Toral, Phys. Rev. E 70, 026112 (2004).

[30] C. P. Herrero, Phys. Rev. E 65, 066110 (2002).

[31] M. B. Hastings, Phys. Rev. Lett. 91, 098701 (2003).

[32] A. J. Bray, J. Phys. A: Math. Gen. 22, L67 (1989).

[33] A. J. Bray, Adv. Phys. 43, 357 (1994).

[34] V. Spirin, P. L. Krapivsky, and S. Redner, Phys. Rev. E 63, 036118 (2001).

[35] V. Spirin, P. L. Krapivsky, and S. Redner, Phys. Rev. E 65, 016119 (2001).

[36] D. Boyer and O. Miramontes, Phys. Rev. E 67, 035102 (2003).

[37] P. K. Das and P. Sen, Eur. Phys. J. B 47, 391 (2005).

[38] O. Häggström, Physica A 310, 275 (2002).

[39] C. Castellano, V. Loreto, A. Barrat, F. Cecconi, and D. Parisi, Phys. Rev. E 71, 066107 (2005).

[40] M. Uchida and S. Shirayama, Phys. Rev. E 75, 046105 (2007).

[41] H. Zhou and R. Lipowsky, Proc. Nat. Acad. Sci. USA 102, 10052 (2005).

[42] C. Castellano and R. Pastor-Satorras, J. Stat. Mech.: Theory Exp. p. P05001 (2006).

[43] C. Godrèche and J. M. Luck, J. Phys.: Condens. Matter 17, S2573 (2005).

[44] C. M. Newman and D. L. Stein, Phys. Rev. Lett. 82, 3944 (1999).

[45] M. Barthélémy and L. A. N. Amaral, Phys. Rev. Lett. 82, 3180 (1999).

[46] M. A. de Menezes, C. F. Moukarzel, and T. J. P. Penna, Europhys. Lett. 50, 574 (2000).

[47] M. E. J. Newman and D. J. Watts, Phys. Rev. E 60, 7332 (1999).

[48] M. E. J. Newman, C. Moore, and D. J. Watts, Phys. Rev. Lett. 84, 3201 (2000).

[49] P. Svenson, Phys. Rev. E 64, 036122 (2001).

[50] P. M. de Oliveira, C. M. Newman, V. Sidoravicious, and D. L. Stein, J. Phys. A: Math. Gen. 39, 6841 (2006). 